\title{
Original
}

\section{Treatment of Boundary Conditions in the Application of Wavelet-Galerkin Method to an SH Wave Problem}

\author{
by

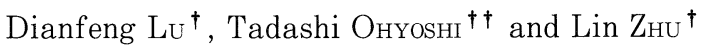

\begin{abstract}
One of main problems with the Wavelet-Galerkin is the treatment of boundary conditions. It is sloved by our method which is referred to as Fictitious Boundary Approach. In this approach, a fictitious boundary is assumed to tackle the difficulty of treating boundary conditions. And an additional condition is also made in order to ensure the real solution. This method is adapted to all the three kinds of boundary conditions in the theory of differential equation. To support our method, an SH wave mode problem in a plate is solved, and its numeral result is compared with the exact solution. Although the Wavelet-Galerkin has very good features for solving differential equations, we find that it is still difficult to represent the infinite resonance solution after investigating the error convergence near the resonance point. And we emphasize the importance of selecting a proper scale $j$ especially to represent the solution near the resonance.
\end{abstract}

Key Words : Computational Mechanics, Wavelet-Galerkin, Differential Equation, SH Wave Problem

\section{Introduction}

The wavelet based approximations of ordinary and partial differential equations (Daubechies, 1992, Glowinski et al., 1994, Qian et al., 1993, and Williams et al., 1992) have been attracting the attention since the orthonormal bases of compactly supported wavelet (Daubechies, 1988) was constructed. And the multiresolution analysis based Fast Wavelet Transform Algorithm (FWT) (Beylkin et al., 1991) has also made the wavelet approximation attractive. Among the wavelet approximations, the Wavelet-Galerkin technique (Amaratunga et al., 1994, Latto et al., 1992, Williams et al., 1994, Williams et al. 1993, and Xu et al., 1992) is the most frequently used because of its simplicity for differential equations. However the difficulty would be encountered because the wavelet based expansion is hard to joint the boundary conditions. Although many efforts (Jawerth et al. 1993, Qian et al. 1993, Williams et al. 1993, and Xu et al. 1992) was made, the difficulty isn't solved essentially. Only the problems that have the periodic boundary conditions or the periodic distributions can be dealt with.

Received March 5, 1996

$†$ Graduate student; Graduate School of Mining and Engineering, Akita Univrsity, Akita, 010 Japan.

$\dagger \dagger$ Department of mechanical Engineering, Mining College, Akita University, Akita, 010 Japan. 
Differing from the above, Williams et al. (July 1994) used polynomial extrapolation technique to tackle the difficulty of settling boundary conditions. And the scaling functions are classified into three kinds. They are Regular Scaling Functions which lies entirely within the boundaries, Irrgular Scaling Function which lies partially inside the interval of boundaries and Exterior Scaling Function which lie entirely outside the interval of boundaries. For the difficulty to deal with the lrregular Scaling Function, they predict the coefficients of Exterior Scaling Function and give a modified form of the Wavelet-Galerkin equations. The method gives a good result, but for the applications to engineering, might be lost its simplicity especially for the Galerkin method.

To solve the difficulty of boundary settlement, we start analysis from a simple SH wave mode problem in a plate. After separating of variables in the governing equation, a one-dimensional ordinary differential equation is gotten. The unknown function $u(x)$ is expanded by scaling functions $2^{j / 2} \phi\left(2^{j} x-k\right)$. For this wave mode problem, the substantial interval is [0, 1$]$, but the expanded functions' support is $\left[\frac{-N+1}{2^{j}}, \frac{N-1+2^{j}}{2^{j}}\right]$. To satisfy the coordinate selection rule of Galerkin method (Mihurin et al., 1968), a fictitious boundary is assumed. In order to ensure the real solution within the substantial interval for the assumed problem, an additional condition is also considered that the solution might satisfy the real boundary condition. Therefore the solution of $u(x)$, which is expanded by a complete coordinate and satisfies both of the governing equation and the real boundary conditions, is unique.

Finally, the numerical result of Dirichlet boundary problem is given to compare with the exact solution and we find a good agreement between them. From the inspection of the error convergence near the resonance, we find that Wavelet-Galerkin method is still difficult to represent the resonance response and the selection of the proper scale of $j$ is very important.

\section{Wavelet Based Complete Coordinate Function}

There are many kinds of wavelet which can be used as complete coordinate functions. Here only the Daubechies Wavelet (Daubechies, 1988) and its several properties are introduced.

Ingrid Daubechies defined the class of compactly supported wavelets. Briefly, let $\phi(x)$ be a solution of the scaling relation

$$
\phi(x)=\sum_{k=0}^{N-1} a_{k} \phi(2 x-k)
$$

The expression $\phi(x)$ is called Scaling Function. And the associated wavelet function $\Psi(x)$ is

$$
\Psi(x)=\sum_{k=-N}^{1}(-1)^{k} a_{1-k} \phi(2 x-k)
$$

Where $N$ is a positive even integral. And $a_{k}$ is a collection of coefficients that have to satisfy the following conditions.

From the normalization $\int \phi d x=1$ of the scaling function, the first condition can be written as follows.

$$
\sum_{k=0}^{N-1} a_{k}=2
$$

The translations of $\phi(x)$ are required to be orthonormal

$$
\int \phi(x-k) \phi(x-m)=\delta_{k, m}
$$


This formula (4) implies the second condition.

$$
\sum_{k=0}^{N-1} a_{k} a_{k-2 m}=\delta_{0 m}
$$

where $\delta$ is the delta function.

Smooth wavelet function requires the moment of the wavelet to be zero.

$$
\int x^{m} \Psi(x) d x=0
$$

This formula (6) implies the third condition

$$
\sum_{k=0}^{N-1}(-1)^{k} k^{m} a_{k}=0
$$

for $m=0,1, \cdots, N / 2-1$.

For the coefficients satisfying with the above conditions, the functions, which consist of translations and dilations of the scaling function $\left(\phi\left(2^{j} x-k\right)\right)$ or the wavelet function $\left(\Psi\left(2^{j} x-k\right)\right)$, form a complete and orthogonal basis. The relation between two functions is expressed as:

$$
V_{j+1}=V_{j} \oplus W_{j}
$$

where

$$
\begin{aligned}
V_{j} & =2^{j / 2} \phi\left(2^{j} x-k\right) ; k=\cdots,-1,0,1, \cdots \\
W_{j} & =2^{j / 2} \Psi\left(2^{j} x-k\right) ; k=\cdots,-1,0,1, \cdots
\end{aligned}
$$

And $j$ is the dilation parameter as the scale. For a certain value of $j$ and $N$, the support of the scaling function $\phi\left(2^{j} x-k\right)$ is given as follows.

$$
\operatorname{supp}\left(\phi\left(2^{j} x-k\right)\right)=\left[\frac{k}{2^{j}}, \frac{N+k-1}{2^{j}}\right]
$$

Figure 1 shows an example of a compactly supported scaling function and its associated wavelet

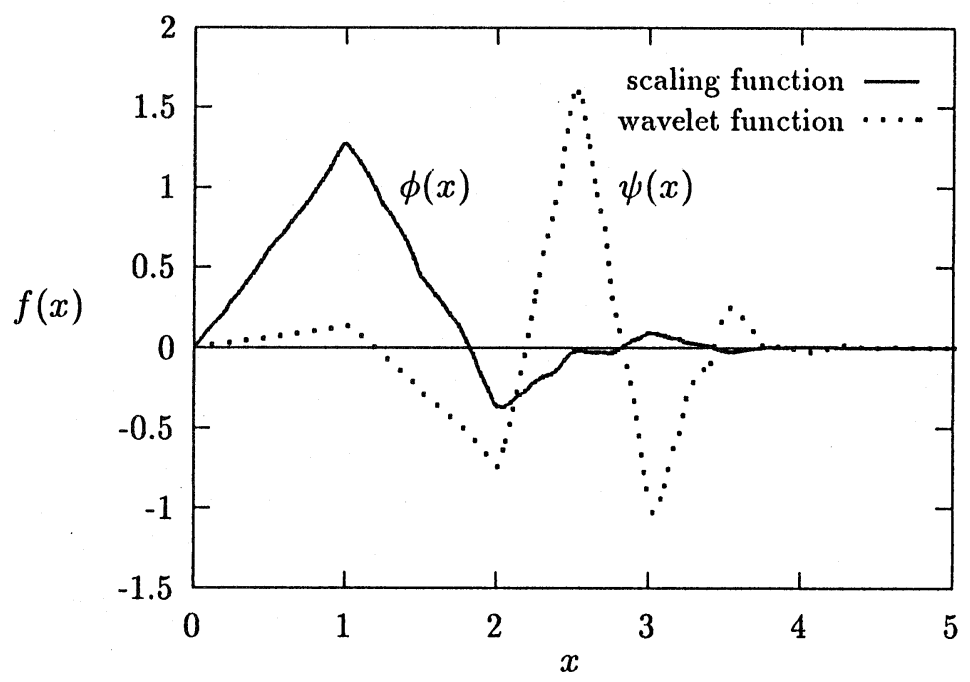

Fig. 1: Daubechies' scaling and wavelet function for $N=6$ 
function with $N=6$.

As the scaling function yields a complete coordinate function basis, it can be used to expand a general function as follows.

$$
f(x)=\sum_{k} 2^{j / 2} c_{k} \phi\left(2^{j} x-k\right)
$$

For this expansion, we have the following convergence property.

$$
\left\|f-\sum_{k} c_{k} \phi\left(2^{j} x-k\right)\right\| \leqq C 2^{-j p}\left\|f^{(p)}\right\|
$$

where

$$
c_{k}=\int f(x) \phi\left(2^{j} x-k\right) d x
$$

and $C$ and $p$ are constants.

Here it is worth emphasizing that selection of a proper scale is very important. For example, to express a function having five periods in one interval, the scale $j$ which at least has five translated components of the corresponding scaling function in the same interval must be selected. (in fact five translated components is still not enough). Besides this, there is another important point that scale $j$ also affects the convergence in computational estimation. As we can see from the convergence property (12), the expanded function approaches the real value of $f$, as $j \rightarrow \infty$.

\section{SH Wave Mode Problem}

Consider a plate of infinite extent in the $z$ and $y$ direction and of thickness 1 in $x$ direction (Fig. 2). For horizontally polarized shear (SH) wave, the governing partial differential equation is:

$$
u_{x x}+u_{y y}=\frac{1 \partial^{2} u}{c^{2} \partial t^{2}}
$$

where $u=u(x, y, t)$ We consider solutions of the wave equation given by

$$
u(x, y, t)=u(x) e^{i\left(\xi_{y}-\omega t\right)}
$$

The substitution of (14) in the wave equation (13) gives:

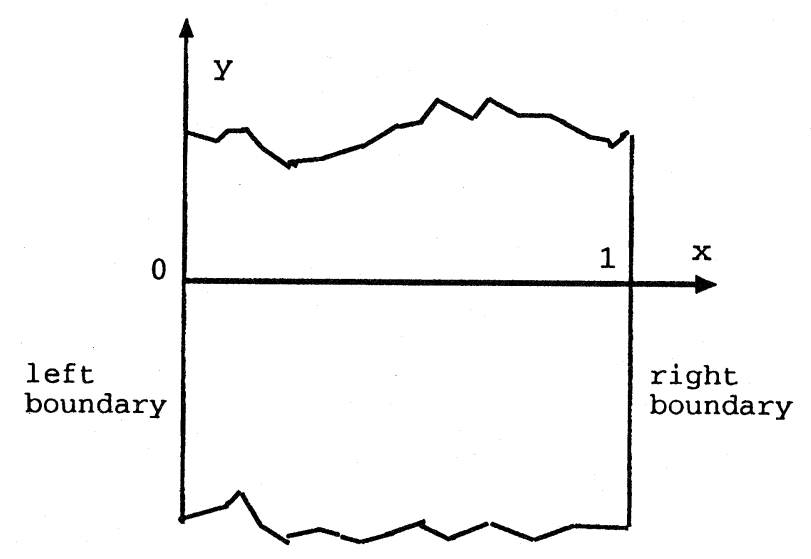

Fig. 2: Coordinate system on an elastic plate of thickness 1 


$$
\frac{d^{2} u(x)}{d x^{2}}+\beta^{2} u(x)=0 \quad, \quad \beta^{2}=\frac{\omega^{2}}{c^{2}}-\xi^{2}
$$

For exact solution we can lead to

$$
u(x, y, t)=\left(A_{1} \sin \beta x+A_{2} \cos \beta x\right) e^{i\left(\xi_{y}-\omega t\right)}
$$

where $A_{1}$ and $A_{2}$ can be decided by boundary conditions.

\section{Wavelet-Galerkin Solution}

For the ordinary differential equation (15), we define the wavelet expansion for unknown function $u(x)$ as

$$
u(x)=\sum_{k=-N+1}^{2^{i}} c_{k} 2^{j / 2} \phi\left(2^{j} x-k\right) \quad x \in[0,1]
$$

where $c_{k}$ are the unknown coefficients. For the problem of Fig. 2, the real and analytical boundaries are originally same. They are 0 and 1 . But the boundaries of the support of the expansion (17) are $\frac{-N+1}{2^{j}}$ and $\frac{N-1+2^{j}}{2^{j}}$ and they have the boundary values $\phi(0)$ and $\phi(N-1)$. In order to use the Galerkin method directly, namely the selected coordinate function has to satisfy the boundary conditions (Mihurin et al. 1968), we reset the analytical boundaries to the boundaries of support for the SH-wave mode problem. This manipulation enable us to use the wavelet based coordinate functi ons. As see, the analytical boundaries are now different from the real boundaries. Then, Iet's say it "Fictitious Boundary Approach" in the wavelet analysis for differential equations.

This approach widens the original boundaries and changes the original boundary conditions to the following boundary conditions.

$$
\begin{aligned}
& u\left(\frac{-N+1}{2^{j}}\right)=\phi(0) \\
& u\left(\frac{N-1+2^{j}}{2^{j}}\right)=\phi(N-1)
\end{aligned}
$$

To ensure to get the correct solution on the interval $[0,1]$, an additional condition is made that the assumed analytical problem has to satisfy the real boundary conditions at point 0 and 1 . As a result, the assumed boundary conditions have no effect on the solution within $[0,1]$. The effected field is only $\left[\frac{-N+1}{2^{j}}, 0\right]$ and $\left[1, \frac{N-1+2^{j}}{2^{j}}\right]$ as we can see later.

Now making the substitution

$$
\begin{aligned}
& X=2^{j} x \\
& C_{k}=2^{j / 2} c_{k}
\end{aligned}
$$

and substituting (17) to the equation (15), we get

$$
\sum_{k=-N+1}^{2^{i}} C_{k} \phi_{X X}(X-k)+p \sum_{k=-N+1}^{2^{i}} C_{k} \phi(X-k)=0
$$

where $p=\frac{\beta^{2}}{2^{2 j}}$

To determine the coefficients $C_{k}$, we take the inner product of both sides of Eq. (20) with $\phi(X-$ $n)$. Usually the integration interval of the product is $[0,1]$. Because the integration interval is narrower than the support, the integration rises a problem that the number of coefficients $\left(C_{k}\right)$ is larger than the number of equations. This is the difficulty of settling boundary conditions for Wavelet-Galerkin method. Because the fictitious boundaries is assumed, the integration interval 
can be widen to $\left[\frac{-N+1}{2^{j}}, \frac{N-1+2^{j}}{2^{j}}\right]$. So the integration interval is the same with the support, and all the translated components is included between the integration interval. As a result the number of coefficients and equations are matched.

$$
\sum_{k} C_{k} \Omega_{n-k}+p \sum_{k} C_{k} \delta_{k, n}=0
$$

where

$$
\delta_{\mathrm{k}, \mathrm{n}}=\int \phi(X-k) \phi(X-n) d x
$$

and the connection coefficients are described in the following. For these coefficients, Latto et al. (1992) gave the exact solutions.

$$
\Omega_{n-k}=\int \phi_{X X}(X-k) \phi(X-n) d x
$$

To meet the additional condition, let the wavelet expansion (17) satisfy the real boundary conditions. Here we only need to consider the scaling functions whose support includes the boundaries, because every scaling functions in (17) only has the contribution coming from its own support field. And we will give the equations of left boundary hereafter, because the equations of right boundary can be solved similarly.

\section{Dirichlet boundary condition:}

This is the simplest one of the three kinds of boundary conditions. The equation can be gotten directly from Eq.(17).

$$
\sum_{k=-N+1}^{2^{i}} C_{k} 2^{j / 2} \phi(-k) \phi(-k)=u(0)
$$

where $u(0)$ is the value of left boundary.

\section{Neumann boundary condition:}

Differing from the Dirichlet boundary, we get

$$
\left.\sum_{k=-N+1}^{2^{i}} c_{k} 2^{j / 2} \phi_{x}\left(2^{j} x-k\right)\right|_{x=0}=u_{x}(0)
$$

For $\phi_{x}(x)$ there

$$
\phi_{x}(x)=\sum_{p} \lambda_{p} \phi(X-p)
$$

where $\lambda_{p}$ is the scaling coefficients of the function $\phi_{x}(x)$ and its exact solution is given by Latto et al. (1992). So that the following relation can be obtained.

$$
\left.\sum_{k=-N+1}^{2^{i}} c_{k} 2^{j / 2} \sum_{p} \lambda_{p} \phi(X-p)\right|_{x=0}=u_{x}(0)
$$

\section{The Mixed Boundary Condition:}

Since the equations for Dirchlet boundary and Neumann boundary can be written as above, the equation for the mixed boundary condition can be expressed as.

$$
\left.\sum_{k=-N+1}^{2^{i}} c_{k} 2^{j / 2} \sum_{p} \lambda_{p} \phi(X-p)\right|_{x=0}+\sum_{k=-N+1}^{2^{i}} c_{k} 2^{j / 2} \phi(-k)=u_{x}(0)+u(0)
$$

From every kind of boundary conditions, two equations can be gotten: one is from left boundary like (23), (26) or (27) and the other from the right boundary. These two equations represent the relation of the coefficients $c_{k}$. Therefore two equations should be eliminated from the system of 
Wavelet-Galerkin equation (21) which also represent the relation of the coefficients $c_{k}$. And this two eliminated equations must, be selected to contain the $C_{k}$ which are included in the above two boundary equations. After eliminating the first and the last equations of Eq. (21), the following matrix form is gotten with $N=6$.

$$
\mathrm{TC}=0
$$

where

$$
\begin{aligned}
\mathrm{C} & =\left[\begin{array}{c}
C_{-5} \\
C_{-4} \\
\vdots \\
C_{2^{j}}
\end{array}\right]_{\left(2^{j}+6\right) \times 1} \\
\mathrm{~T} & =\left[\begin{array}{ccccccccc}
\Omega_{1} & \Omega_{0}+p & \cdots & \Omega_{-4} & \cdots & 0 & \cdots & 0 & 0 \\
\vdots & \vdots & \vdots & \vdots & \vdots & \vdots & \vdots & \vdots & \vdots \\
\Omega_{4} & \Omega_{3} & \cdots & \Omega_{-1} & \cdots & 0 & \cdots & 0 & 0 \\
0 & \Omega_{4} & \cdots & \Omega_{0}+p & \cdots & 0 & \cdots & 0 & 0 \\
\vdots & \vdots & \vdots & \vdots & \vdots & \vdots & \vdots & \vdots & \vdots \\
0 & 0 & \cdots & 0 & \cdots & \Omega_{3} & \cdots & \Omega_{0}+p & \Omega_{-1}
\end{array}\right]_{\left(22^{j}+4\right) \times\left(2^{j}+6\right)}
\end{aligned}
$$

Now the combination of Eq. (28) with two equations from boundary conditions gives a linear algebraic equation of $C_{k}$ which can be easily solved by a conventional way such as the Gaussian elimination algorithm. Consequently the solution of $u(x)$ can be obtained directly from equation (17) with the substitution of $C_{k}$.

\section{Numerical Result}

We solve the equation (15) with $\mathrm{N}=6$, and compare its numerical result with the exact solution (16). Figure 3 is the $u(x)$ distribution in the interval $\left[\frac{-N+1}{2^{j}}, \frac{N-1+2^{j}}{2^{j}}\right]$ with $j=7$. In the interval $[0,1]$, both results for $\beta=3.5 \pi$ and $\beta=9.5 \pi$ show good agreement with the exact solutions. And we can find that there is no effect from the fictitious boundaries on the solutions to be obtained.

To explain the importance of the selection of $j$, a very big value of $\beta$ and a small value of $j$ are selected. The trial computation for $\beta=15.5 \pi$ and $j=4$ yields the high frequency vibration solution which is bad agreement with the exact solution (Fig. 4). It means that $j=4$ is not enough for the exact solution at high frequency.

To show difficulty in computation with Wavele-Galerkin method, that arises around the resonant frequencies of the system, examples for $q=2.999999, j=10$ are demonstrated in Fig. 5 . The amplitude and the direction of vibration are all different with those of exact solution.

For this problem, the resonance condition is

$$
\beta=\pi q, \quad q=0, \pm 1, \cdots, \pm n, \cdots
$$

To investigate the computation around the resonance point, we draw the relative maximum error $\left(\hat{e}_{\max }\right)$ distribution graph at neighboring points approaching to the resonance point.

$$
\hat{e}_{\max }=\frac{\left|u_{w}-u_{e}\right|_{\max }}{u_{e}}
$$

where $u_{w}$ is the wavelet solution and $u_{e}$ is the exact solution.

Figure 6 shows clearly that the relative maximum error become higher as $q$ goes near 4 . It 


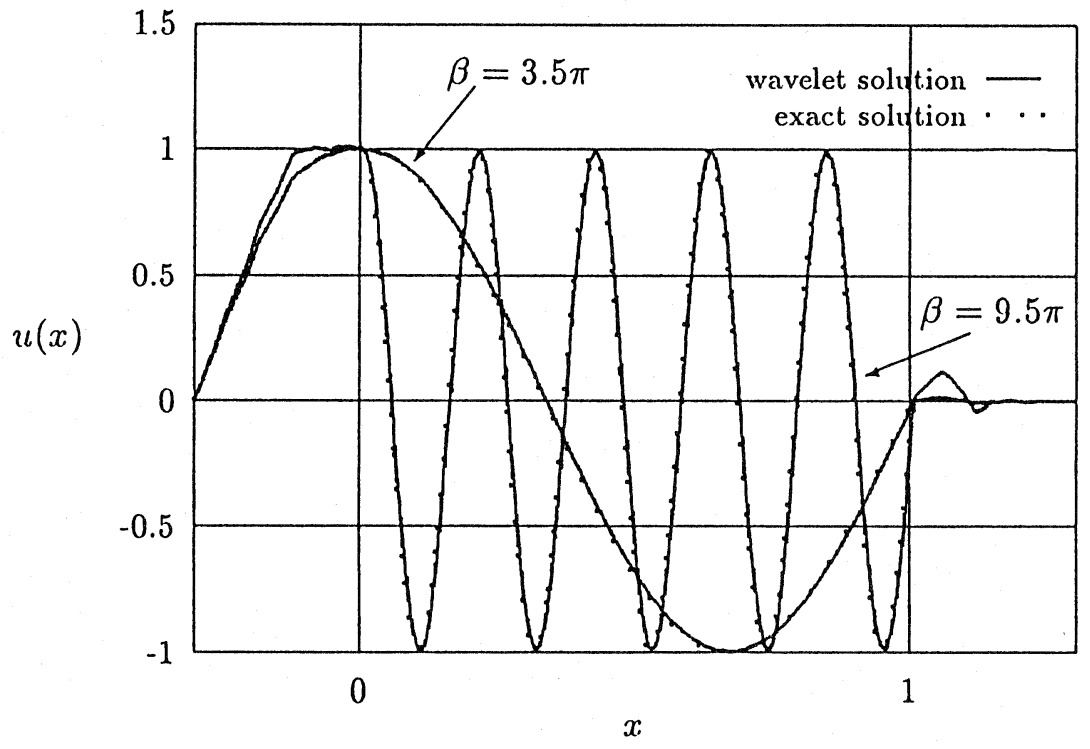

Fig. 3: Behavior of $u(x)$ within the interval $\left[\frac{-N+1}{2^{j}}, \frac{N-1+2^{j}}{2^{j}}\right]$

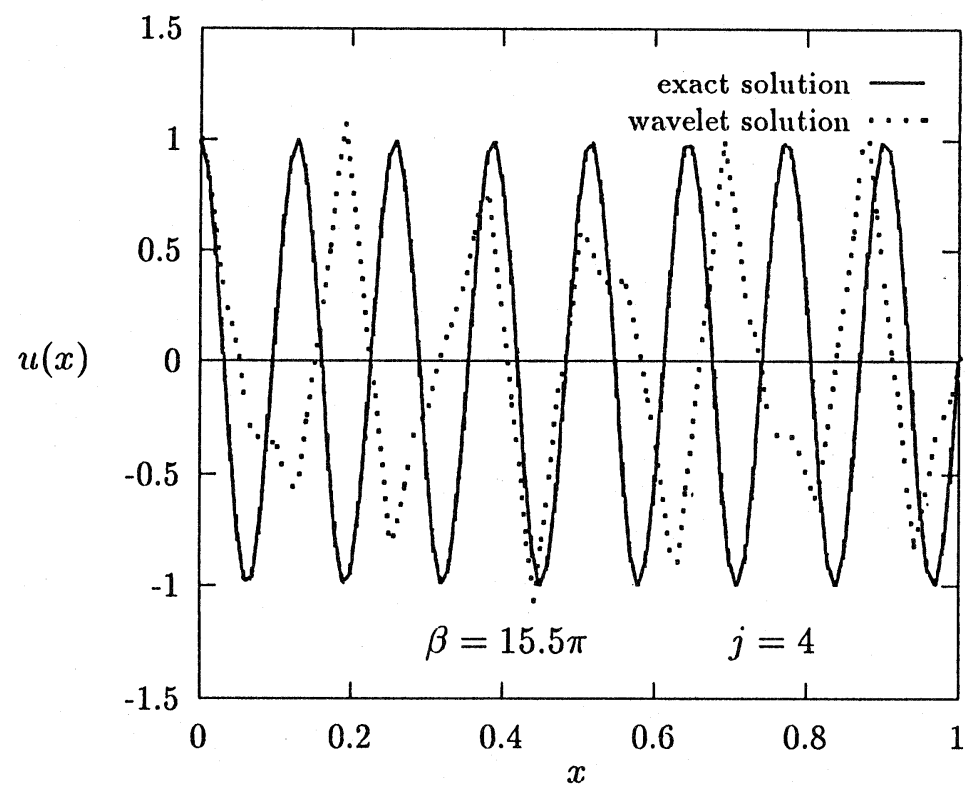

Fig. 4 : Example of the bad result $u(x)$ for improper value of $j$ 


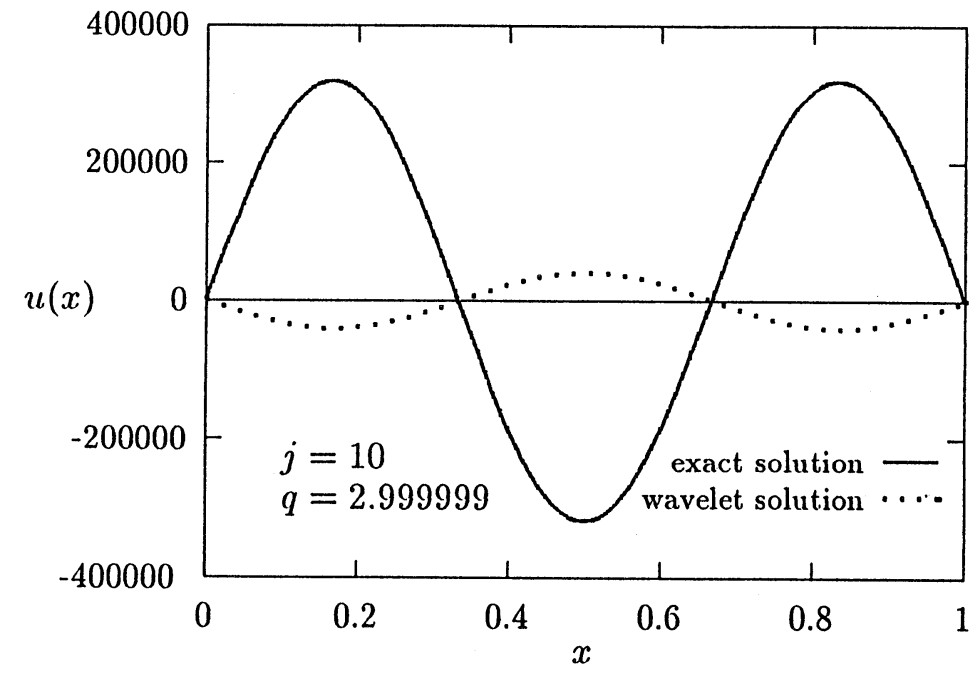

Fig. 5 : Example of the bad result of Wavelet-Galerkin solution near the resonance

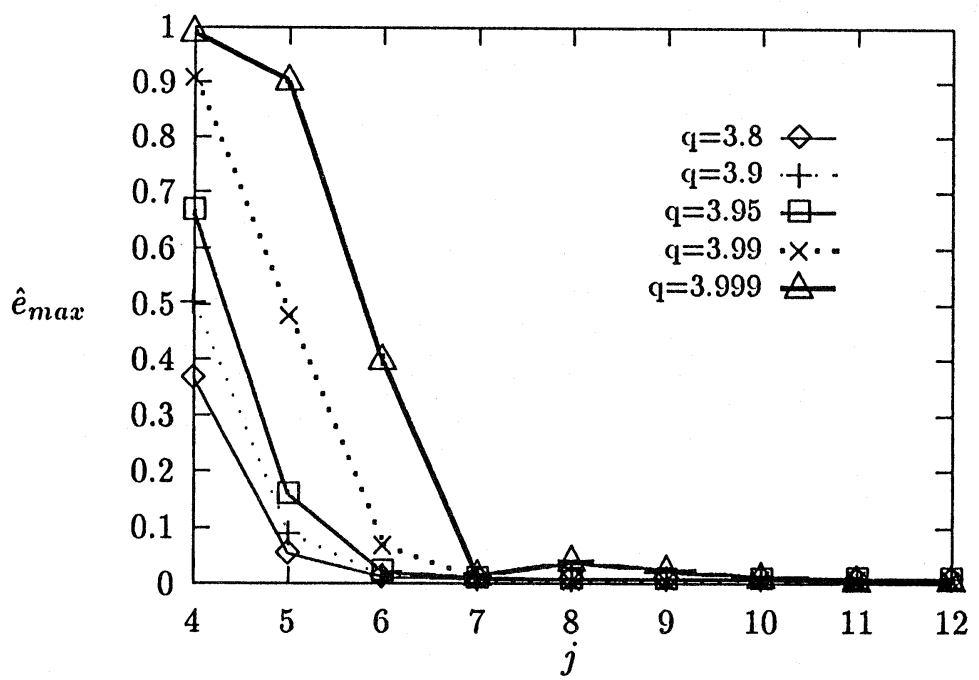

Fig. 6: The relative maximum error variation with $j$ near the resonance point

means that in order to represent the real solution, the nearer to the resonance points, the bigger value of $j$ is needed.

For this phenomenon, we explain as follows. Since the solution $u(x)$ of a certin point is made up of the summation of wavelets, the bigger the value of the point is, the more number of wavelets is needed. As we know from Eq. (10), the $j$ is bigger, the more wavelets can be managed. 
Therefore to represent the very big value of resonance solution, a very big value of $j$ is also required. But in above explanation, we ignore the working of $c_{k}$. As we know from Eq. (17), the combined effect of scaling function $\phi(x)$ and coefficients $c_{k}$ decides the amplitude of $u(x)$. But here when the solution $u(x)$ goes bigger, the coefficients $c_{k}$ don't become bigger, instead the big $j$ is required to get more wavelet. This problem needs further research.

As the $j$ goes bigger, the another problem will be brought out that we have to solve a very big simultaneous equation. For example, when $j=12$, we need to solve $2^{12}=4096$ simultaneous equations. As a result, it is troublesome for Wavelet-Galerkin method to represent such quasi-resonant solutions.

Excepting the bad informations from the Fig.6, we can read out good information that the Wavelet-Galerkin method has very fast convergence in a good many ordinary case. This point has been well examed by Amaratunga et al. (1994). And Fig. 6 also gives the standard of selecting a pr oper scale $j$ for $N=6$ wavelet. For usual condition, $j=6$ is a proper value for enough accuracy an $\mathrm{d}$ we have no big simultaneous equations $\left(2^{6}=64\right)$ to be calculated.

\section{Conclusions}

The Wavelet-Galerkin method has been shown to be a powerful tool for the fast and accurate solution of differential equations. For the difficulty of treating boundary conditions, we offered the idea of a fictitious boundary that the Galerkin method can be used directly. And the original boundary condition is changed to be an additional condition to ensure to get the real solution on the real interval $[0,1]$. This can be adapt to all the three kinds of boundary conditions. The good agreement of its numeral result with the exact solution proves its availability. Then, we call it the Fictitious Boundary Approach.

Finally, the inspection of the error convergence with $j$ was made by the computation near the resonance point (Fig. 6). Then, it looks difficult for the present to analyze the quasi-resonance response by the Wavelet-Galerkin method. The selection of a proper value of scale parameter $j$ becomes very important especially for representing the solution near the resonant points. However, $j=6$ is a proper value of the scale for usual condition except at the resonant point.

\section{References}

Amaratunga, K., and Williams, J. R., 1994, Wavelet-Galerkin Solutions for One-Dimensional Partial Differential Equaions, Inter. J. Num. Meth. Eng., 37, 2703-2716.

Beylkin, G., Coifman, R., and Rokhlin, V., 1991, Fast Wavelet Transforms and Numerical Algorithms I, Comm. Pure Appl. Math., 44, 141-183.

Daubechies, I., 1988, Orthonormal bases of compactly supported wavelets, Commun. Pure Appl. Math., 41, 909-996.

Daubechies, I., 1992, Ten Lectures on Wavelets, Society for Industrial and Applied Mathematics, Philandelphia, Pennsylvania.

Glowinski, R., Lawton, W., Ravachol, M., and Tenenbaum, E., 1990, Wavelet solution of linear and nonlinear elliptic, parabolic and hyperbolic problems in one space dimension, Proc, 9 th Int. Conf. Num. Meth. Appl. Sci. Eng., SIAM, Philadelphia.

Jawerth, B., and Sweldens, W., April 1993, Wavelet multiresolution analyses adapted for the 
fast solution of boundary value ordinary differential equations, Proc, 6 th Cop. Mount. Multi. Conf.

Latto, A., Resnikoff, H., and Tenenbaum, E., 1992, The evaluation of connection coefficients of compactly supported wavelets, proc. French-USA workshop on Wavelets and Turbulence, Princeton Univ., June 1991, Springer, NY.

Mihurin, C. Г., Shimoritukii, X. JI., Tutui, T. T., (translater), 1968, bibun zekibun hoteisiki no kinji kaiho, sogo tosyo, 153-288. (In Japanese)

Qian, S., and Weiss, J., 1993, Wavelets and the numerical solution of partial differential equations, J. Compu. Phy.,106, 155-175.

Williams, J. R., and Amaratunga, K., Oct. 1992, Introduction to wavelets in engineering, IESL Tech. Rep. No. 92-07, Intelligent Engineering Systems Laboratory, MIT.

Williams, J. R., and Amaratunga, K., 1993, Wavelet based Green's function approach to 2D PDEs, Eng. Compu., 10, 349-367.

Williams, J. R., and Amaratunga, K., July 1994, High order wavelet extrapolation schemes for initial problems and boundary value problems, IESL Tech. Rep. No.94-07, Intelligent Engineering Systems Laboratory, MIT.

Xu, J. C., and Shann, W. C., 1992, Galerkin-wavelet methods for two-point boundary value problems, Numer. Math., 63 123-144. 